\title{
7 \\ Generating Abstract Datatypes with Remote Access Capabilities
}

\author{
Martin Schuetze, Bernd Schuermann, Joachim Altmeyer \\ University of Kaiserslautern, Germany
}

Abstract:

We propose a software system to specify data management components of CAD tools on the modelling level. The implementation of the resulting abstract datatype will be done by a set of generators. Functions to access data stored outside the abstract datatype are also generated. The information models of the ADT and the external data may differ; the transformation between both can be defined in a mapping table and will be handled by the generated code.

\section{Introduction}

An important aspect of ECAD framework technology is to provide efficient and easy-to-use access to design data for the different tools. While design tools can be supplied with a limited amount of data (for the current design task) in a single transfer, e.g. via files, design management or analysis tools potentially need access to all design data stored by the framework. Usually, this access is provided through a library of access functions to extemal data sources (e.g. databases). The programmer of management tools or design tools has to implement code for the data structures the tool works on as well as code to translate between the tool's data structures and the information model of the accessed library.

We investigated the structure of the design tools in the framework of our PLAYOUT VLSI Design System Zim89, SAP94/. All tools are structured as it can be seen in figure 1. The data needed by a tool are stored in an abstract datatype (ADT). The graphics component, if part of the tool, displays data stored in the ADT. The control component determines the internal control flow, while the user interface lets the designer interact with the tool. The investigation showed that all shaded components are very similar from tool to tool. For example, the ADTs of different tools manage different data models, but the way objects are accessed is the same in all ADTs. Because of their supporting nature for the design algorithms we call these components framework components. The algorithms are highly individual. But while the development of algorithms is a creative task, a lot of time is needed to implement and test the other components which is lost for the algorithm development.

Our approach to overcome these problems is to specify the framework components on the modelling level and let the implementation be done by a generator. Because this work is most advanced in the field of data modelling, we will consider only this aspect in the following sections. For the data management components, we can specify a mapping between local data structures and external data structures in addition to the information model. A set of generators creates the corresponding ADT, including methods to access data stored outside the tool. 


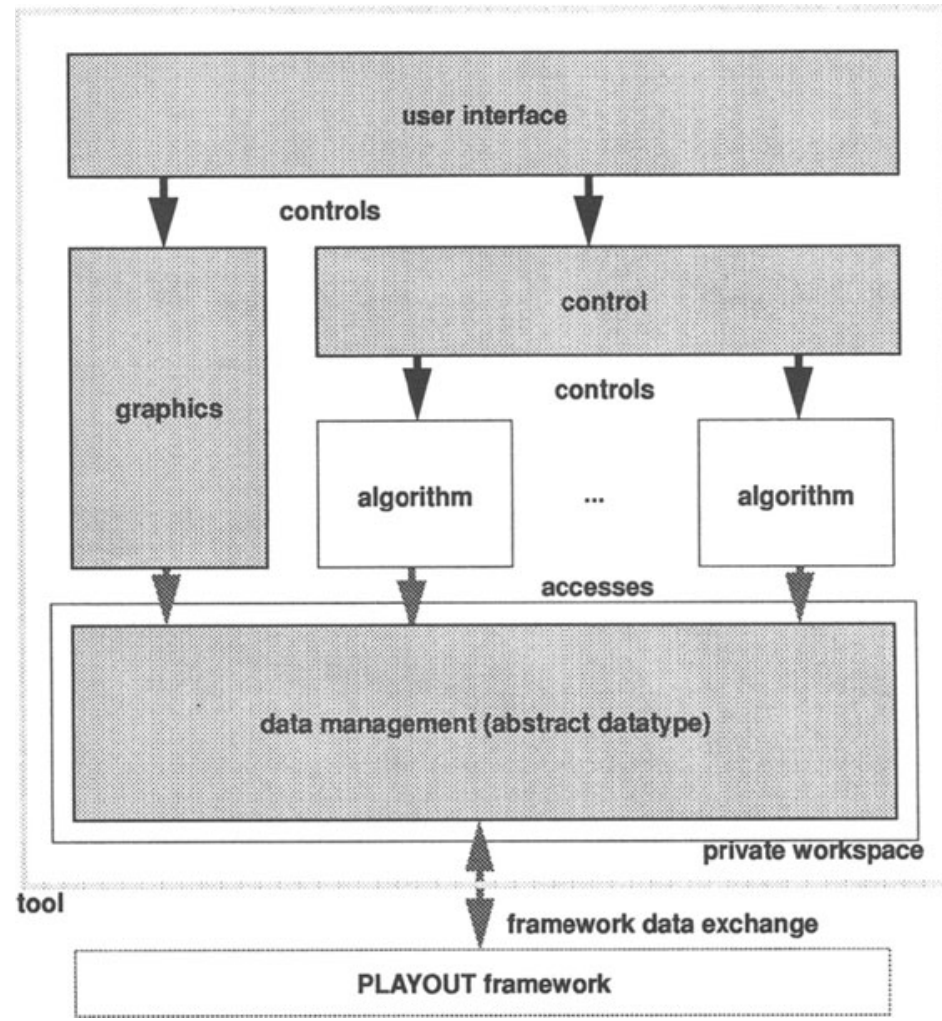

Figure 1 Architecture of a Tool

\section{Data Management within the PLAYOUT Framework}

The PLAYOUT framework, like many others, consists of several tools grouped around a database which holds the design data (see figure 2). We will refer to the database as the archive workspace or the server. The design tools (the algorithms) themselves do not work on the database directly. They access their data in the private workspace (the client) via a programming interface of the generated ADT. The private workspace holds the local tool data and can be seen as a cache of the server data. The data are transferred via files or via socket communication between the server and the clients. A data exchange between private workspaces is also possible. In this case, one workspace acts as a server to the other private workspace. The data for the clients are generally accessed in a check-out-check-in manner. All transaction, consistency, and version control is done within the server and trans- 


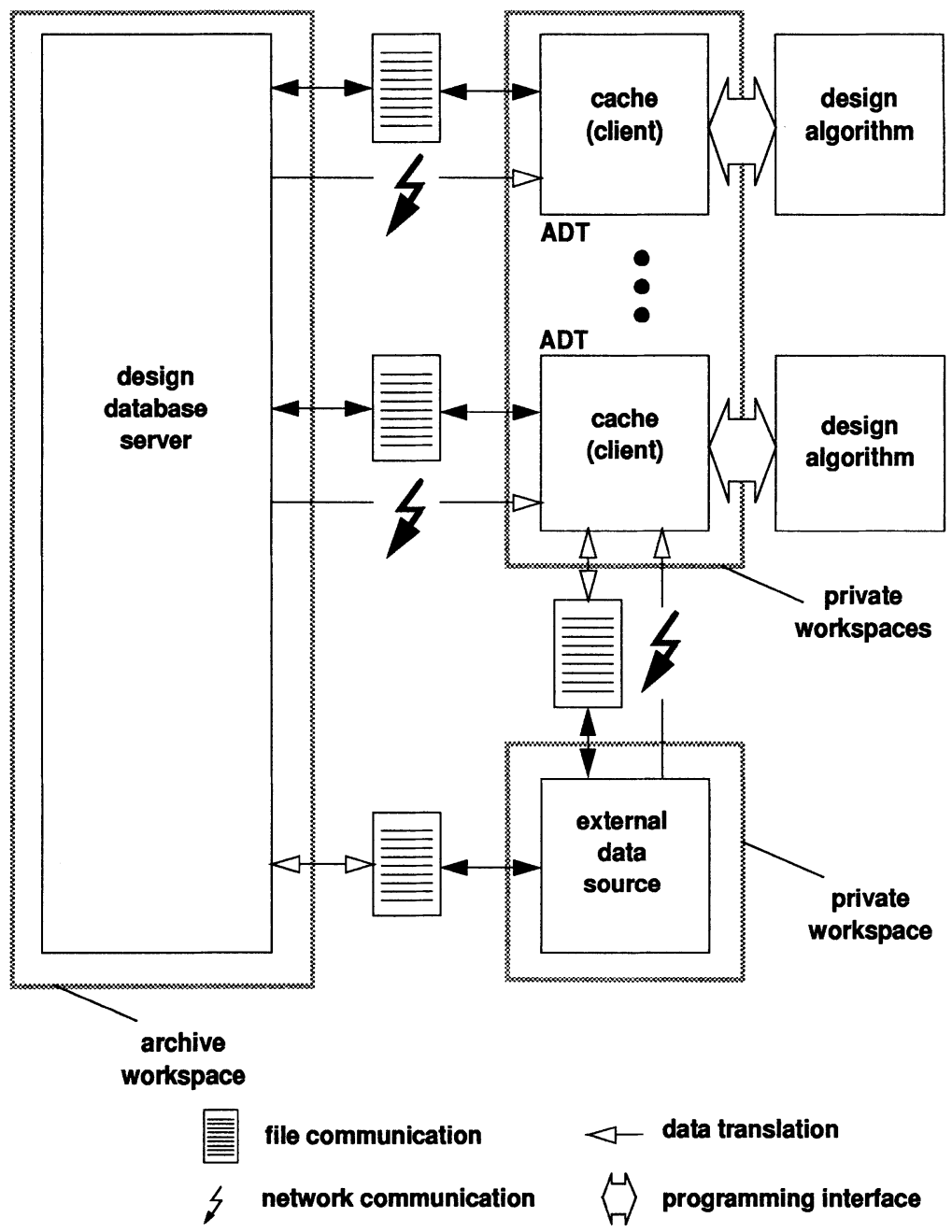

Figure 2: Data Management in the PLAYOUT framework

parent to the tools. The data exchange between client and server is managed by the workspaces and not visible by the tools. The data management described here acts as a infrastructure which is exploited by the generated code for the framework components. 


\section{MOOSE}

The modelling of framework components as well as the code generation is done within the MOOSE system (Model-Based Object-Oriented Software Generation Environment). This section describes the development of appropriate models as well as the structure of MOOSE and the generated code.

\subsection{Models}

All framework components have to be described by models which are input to the generators. Our current implementation of MOOSE allows the graphical definition of information models with extended entity-relationship-diagrams (EER). The development process of the information models is divided into two steps. In the first step, a base model describing the whole application domain of the framework (in this case ECAD) has to be found. This idea is shared by many approaches to model the VLSI design process /HRS92, BrK92, CFI92/. However, this base model is often not very well suited for the implementation of tools. It usually covers more aspects of the framework than a single tool needs. In addition to that, most tools need local data structures not contained in the base model. We therefore modify the base model in a second step with tool specific characteristics to achieve the application model which describes all data needed for the tool.

\subsection{MOOSE architecture}

The architecture of MOOSE can be seen in figure 3. The user enters the base model and the characteristics of an application with the graphical editor. The editor checks that only syntactically correct information models are entered. The information models are kept in an ADT (which has been generated by MOOSE, too). Several generators are provided to generate different types of output: The $C$ generator produces an ADT of the application model in the programming language $\mathrm{C}$. The $\mathrm{C}++$ generator produces a $\mathrm{C}++-\mathrm{ADT}$, respectively. The network communication generator allows access to external data sources in conjunction with the $\mathrm{C}++$ generator. It is described in more detail in section 4. The Versant generator creates code for the commercial OODBMS Versant. The hypertext generator creates FrameMaker hypertext documents for the tool programmer with an implementation description of the information models. The file communication generator generates code to save and restore data of the ADT to/from disk. These files can either be used to checkpoint applications or to exchange data between different ADTs. More generators for different framework components (graphics, user interface) are under development.

\subsection{Generated code}

All ADT code generators offer nearly the same functionality for the tool programmer. They create the following methods/functions for operations on the objects:

\section{- Object creation}

- Object deletion

If an object is deleted which is still referenced by other objects, the reference will destroyed. Undefined references are avoided.

- Attribute access functions Attribute values can be set or read.

- Relation access function 


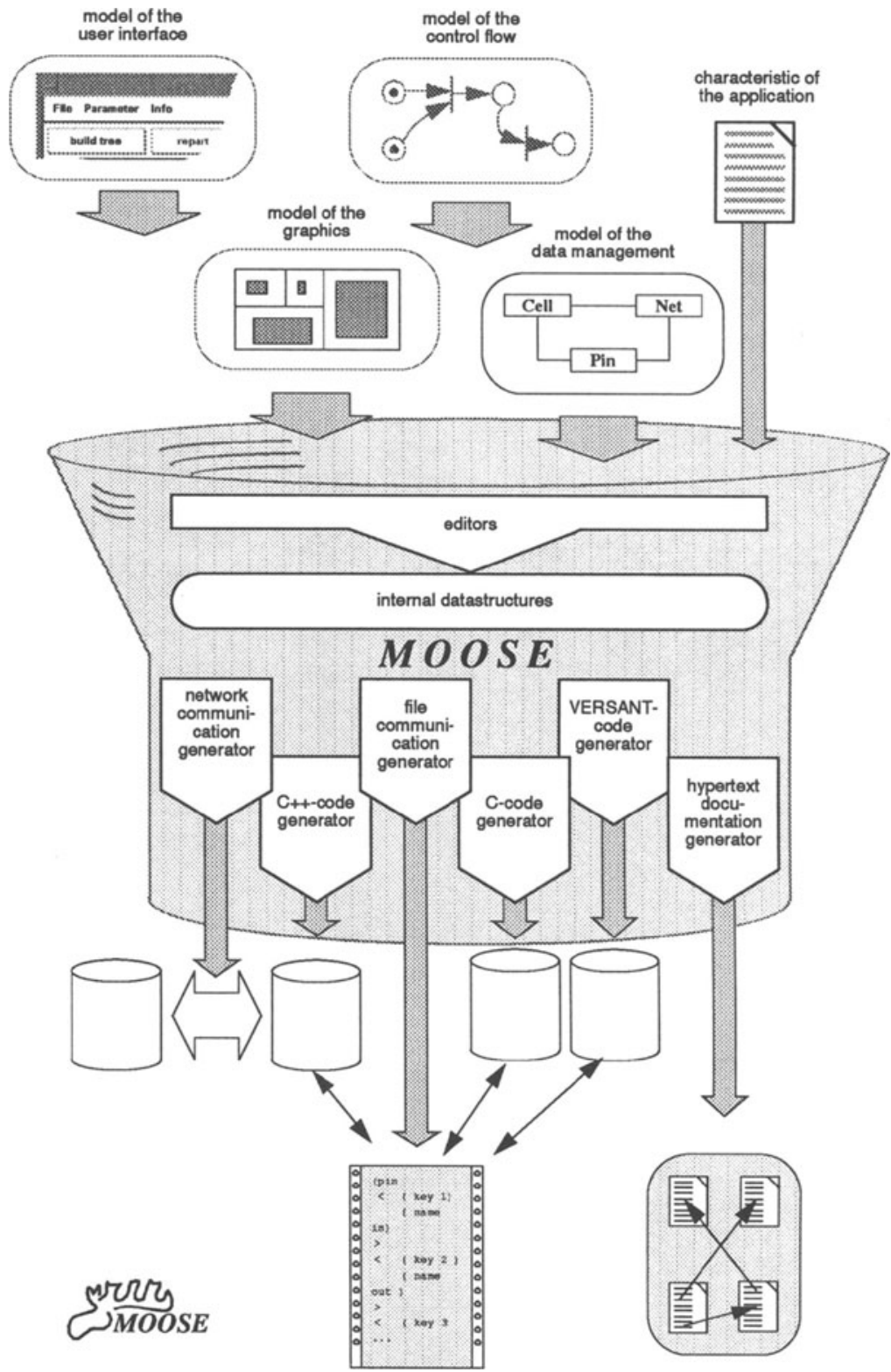

Figure 3: MOOSE System 
Relations between object instances can be established or deleted. The objects participating in a relation can be retrieved from the object holding the relation.

- Checkpointing

Methods are provided to save the state of the workspace to disk or to restore data from disk.

- Meta informations

Objects can be queried about their type and about the relations and attributes provided by this type. Access methods can be sent dynamically at runtime to these objects by a string-to-method-mapping.

\section{- Remote access capabilities}

The ADT can remotely access data stored outside the private workspace. At the moment, only read access to these data sources is allowed. Write access will be added in the future. Differences in the local and the remote information model can be described in mapping tables and are also handled by the generated code.

- Other operations

There are a number of other operations defined on objects, types, and workspaces such as pretty printing and workspace initialization.

All generated classes can be further extended by the user. Methods can be added to the ADT in additional files. They will not be overwritten if the ADT is generated again.

Our approach to the generation of workspaces has some similarities with the generation of database schemata, particularly for object-oriented databases /ODM93/. However, our framework architecture provides one single, central design database. Most tools aren't and shouldn't be implemented on top of a database directly. They work on local data structures only and don't need database support as multi user support, transactions, or atomicity during execution. The MOOSE system provides a workspace functionality and 'comfort' for the tool programmer as some databases do, but without the overhead usually introduced through the database concepts. As stated above, these database concepts are provided by the server. However, if a single tool needs direct database support, this can be achieved with the Versant code generator.

\section{Remote Access Capabilities}

Workspaces created by the $\mathrm{C}++$ generator can exchange data via two different file formats: PLIF and SLANG. SLANG (Simple Data Description Language) provides a general structure to describe objects, attribute values, and relation values. The exact file format is defined during the generation process of the ADT and can be used to store and restore the workspace contents efficiently, as well as to exchange data between generated ADTs. PLIF (Playout Interchange Format) is a defined file format (similar to EDIF) for the exchange of data between all design tools. Most of our design tools access data through PLIF because it is an easy and efficient way to transfer all data needed for one design step. All data needed for one of these tools can be determined a priori by the server and can be retrieved in one single query which makes the transfer process easy and efficient.

However, for some tools we can make no assumptions about the data they need at runtime. Design management and analysis tools potentially need access to all design data stored in the server. It is more efficient if they retrieve their data dynamically during runtime from the server. This is often achieved through libraries allowing to access, for example, databases by using SQL statements, leading to the following problems:

- The information models of the ADT and the external data source do not match. The programmer of CAD tools is forced to translate between the different data structures.

- The programming language and the retrieval language may differ.

- The library is not updated automatically in case that the information model for either the server or the cli- 
ent are changed.

Our network communication generator partially overcomes these problems by generating the necessary methods for remote access along with the other ADT methods. One important goal is to keep the access as transparent as possible. Ideally, the tool programmer 'sees' only the tool's application model and doesn't need to know about the server's information model.

\subsection{Architecture}

The generated access methods are part of a client server architecture as it can be seen in figure 4. An access to

archive workspace (server): private workspace (client):

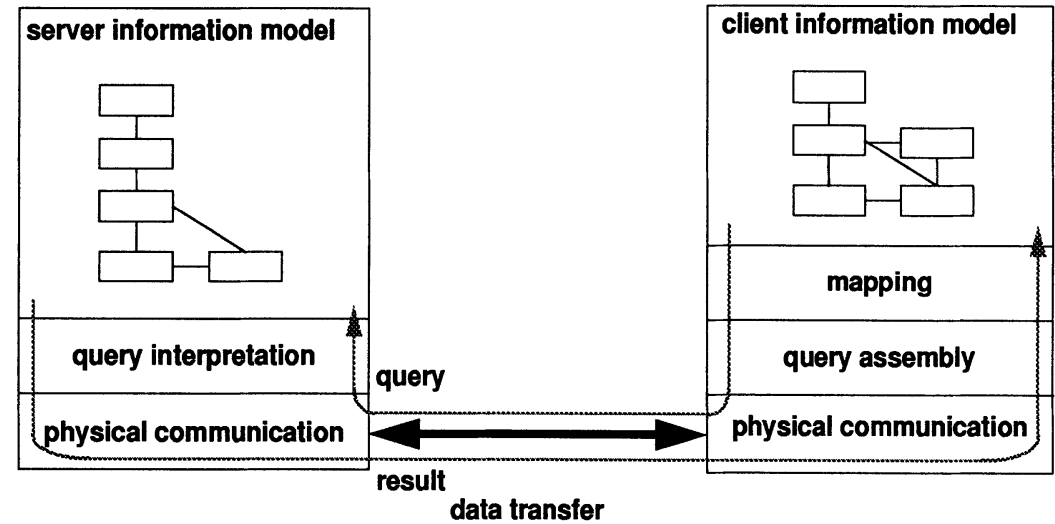

Figure 4: $\quad$ Remote Access

attributes or relations of the client ADT is handled by generated methods. If a remote access is necessary, the attribute/relation of the addressed client object is mapped by these methods to the corresponding server object's attribute/relation. A retrieval string is created to access the required relation/attribute on the server side. The physical communication layer transfers the query to the server where it is interpreted. The result is transferred back into the client and, after conversion to the client's data structures, delivered as the result of the originally called client access method. At the same time, the accessed data are cached for later use.

In order to establish the remote access service in the private workspace, we need:

- A communication link between client and server

We have chosen the UNIX socket mechanism. Other techniques could also be used, e.g. RPC libraries.

- A query language

Objects must be accessed in the server. This is achieved through a query language. Strings over this language must be composed at the client side and interpreted at the server side. We use the query language of our design database.

- A key to identify objects uniquely on both client and server side

The key must be defined on all client objects and on all server objects accessible by the client. It may be a simple number or a pointer as well as complex string. We have chosen a hierarchical key composed with respect to the object (partOf-) hierarchy of the design objects. 


\subsection{Mapping between two information models}

As stated above, our generator is capable of generating code which has a limited capability to map between different information models on client and server side. It is obvious that not all differences between two models can be handled by this generator. In this subsection we will explain which types of mapping can occur within our framework and which of them can be handled by our generator.

Because the mapping can not be derived from the client information model, it has to be defined in a mapping table. This table may contain an entry for every attribute and every relation of all object types. There are three ways to specify the mapping between client and server models: the client objects's attribute/relation name is mapped to a name on the server side (name-to-name-mapping). Or the client's name is mapped to a complex query for the server (name-to-query-mapping). A third possibility is to specify C++-Code which is put into the generated code by the generator (complex mapping). This is the most 'manual' mapping but it allows to deal with nearly arbitrarily complex mapping problems.

\section{Attribute mapping}

The following cases can occur while accessing attributes:

- The object type in the server's information model might have more attributes than the corresponding object type in the client's information model.

We simply ignore these additional informations.

- The object type in the server's information model might have less attributes than the corresponding object type in the client's information model.

We solve this problem by assigning initial values to attributes not available on the server side.

- Attributes may have different names in different information models. We map the client object's attribute name to the server object's attribute name (name-to-name mapping).

- Attributes are stored in other server objects.

This case may appear if the server information model supports more object types than the client, and the client combines these server objects types to one client object type. If the object holding the attribute can be accessed unambiguously from the current client object, a simple query expression can be used to map the attribute (name-to-query mapping). Otherwise, the mapping must be done with a $\mathrm{C}++$-statement (complex mapping).

\section{Relation mapping}

Basically, the relation mapping works exactly as the attribute mapping. However, there are two more problems which can arise:

- Relations may have different cardinalities.

If the cardinality of the relation on server side is a subset of the cardinality of the relation on the client side the mapping can be handled with a name-to-name mapping. If there are more objects in the server object's relation than in the client object's relation and a subset can be selected at every time, either a query expression or a C++-statement can be used for the mapping. All other cases need manual interference after the generation of the access functions.

- Relations may only exist on either client or server side.

If a missing relation in the server can be substituted by the evaluation of a set of other relations, the access function can again be generated by using name-to-query mapping. If such a query cannot be found for a certain relation, it is not possible to generate remote access code for it. 


\section{Object mapping}

A mapping between groups of objects (split or merge of object types) is currently not supported by the generator. These problems must either be solved by mapping each attribute or relation individually as stated above (which is sufficient in many cases). Or it must be solved on the modelling level, e.g. by creating the appropriate object types in the application model.

\subsection{Current Implementation}

Our current implementation of the network communication generator allows the definition of application models and mapping tables with MOOSE as stated above. The access functions are generated as declared in the mapping table. We currently allow only remote read access until a new multi-user synchronization technique is fully implemented on the server side. However, write operations are already supported by the transportation layer and our query language.

An application model may contain parts which access the server as well as parts managed only locally. This can be determined on a per-relation or per-attribute basis. Once the ADT is generated, the remote access is nearly invisible to the tool programmer. However, if the programmer wants to, he may manipulate the caching scheme as well as he may inquire if an object is created locally or remotely accessed. If the server or client information model is changed, all depending ADTs can be consistently updated by modifying the mapping table and re-generating and recompiling the ADT code. Although the definition of complex mappings requires some manual work, it is still helpful because it allows the generation of the access method body and automatically inserts the complex mapping.

\section{Related Work}

OODBMS grant transparent access to objects in distributed repositories. At least databases conformant to the ODMG /ODM93/ standards allow the generation of ADTs from an information model. Although our description and generation techniques are similar, we can not use this type of database directly. We believe that design data management in a framework is the task of the central database and not a tool issue. Tools within the PLAYOUT framework are therefore created without direct database support at runtime.

As far as we know, OODBMS do not support a transparent access to data stored in different databases with different information models. Chomicki and Litwin/ChL94/ describe a possibility of object mapping between different databases by an extension to the OSQL language. They distinguish 16 different types of mapping. Many of these mapping types are similar to the mappings we propose. However, they concentrate on multi-database mappings only, therefore, their approach can not be used directly.

Newer techniques for the management of distributed objects (e.g. OMG /OMG92.1, OMG92.2/, DCE /RKF92 $\int$ allow to access objects distributed over a network of heterogenous computers. However, they don't support the programmer with an automated mapping between different organizations of data. It is usually neither possible do define and generate an ADT, nor are the problems of different information models addressed. However, we intend to use these techniques for the communication layer in the next version of our generator.

Software Engineering Environments allow the definition of information models, object hierarchies, and dynamic aspects of an application. However, the description techniques used are intended to document the specification of components and are often too general to generate ready-to-use application code. We do not know of any SE environment supporting our concepts of base models, application models, and framework components. 


\section{Conclusion}

We showed that it is possible and feasible to specify and generate ADTs with remote access capabilities. Through the principle of generation, we can always guarantee a consistent set of ADTs derived from the same information base model. The techniques described in this paper are currently used in different applications of our CAD framework. The access to other workspaces is nearly transparent to the tool programmer. Because of the simplicity of the transportation layer and the query language, other servers than databases (e.g. other tools) could be used. This could support cooperative strategies between private workspaces.

MOOSE and its generators can be used in many applications. Once the base model is defined, a great amount of work is saved with every new tool introduced or with every change to the data model. Measurements within our framework show that the data management components make up $25 \%$ to $40 \%$ of the tool code. Generating this code saves implementation time and makes MOOSE useful for 'normal' application development as well as for rapid prototyping or the development of experimental systems. The generation of remote access methods is not intended as a replacement for database technology but as an alternative way to load the private workspace with data; the tools still process data locally.

In the future, we will add new capabilities as automatic object key management, remote write access, automatic propagation of data changes, and more support for the mapping between information models as soon as we develop the proper relation semantics.

And finally, the integration of models and generators for the other framework components (like the user interface or the control component) will enable us to generate a complete tool skeleton with MOOSE, leaving the creative part for the tool programmer: the development and implementation of design algorithms.

\section{References}

/BrK92/ M. Brielmann, E. Kupitz, "Representing the Hardware Design Process by a Common Data Scheme", Proc. Int. European Design Automation Conference, 1992

/CFI92/ "Design Representation Electrical Connectivity Information Model and Programming Interface", CFI Pilot Release Document, CFI-92-P-6, 1992

/ChL94/ J. Chomicki, W. Litwin, "Declarative Definition of Object-Oriented Multidatabase Mappings", in M. T. Ozsu, U. Dayal, P. Valduriez, "Distributed object management", Morgan Kaufmann Publishers, 1994

/HRS92/ C. Huebel, D. Ruland, E. Siepmann, “On Modeling Integrated Design Environments”, Proc. Int. European Design Automation Conference, 1992

JODM93/ T. Atwood, J. Duhl, G. Ferran, M. Loomis, D. Wade, "The Object Database Standard: ODMG-93", edited by R.G.G. Cattell, Morgan Kaufmann Publishers, 1993

/OMG92.1/ R. M. Soley (ed.), "Object Management Architecture Guide", Rev. 2.0, OMG TC Document 92.11.1, John Wiley \& Sons, 1992

1OMG92.21 "The Common Object Request Broker: Architecture and Specification", Rev. 1.1, OMG Document 91.12.1, John Wiley \& Sons, 1992

/RKF92/ W. Rosenberry, D. Kenney, G. Fisher, “Understanding DCE”, O’Reilly \& Associates, 1992

ISAP94/ B. Schurmann, J. Altmeyer, H. Pahle, M. Schutze, "A Framework for the VLSI Design System PLAYOUT", GI/TTG/GME-Fachtagung "Rechnergestutzter Entwurf und Architektur mikroelektronischer Systeme", Oberwiesenthal, 1994 (in German)

Zim89] G. Zimmermann, "PLAYOUT - A Hierarchical Design System", Information Processing 89, G.X. Ritter (ed.), Elsevier Science Publishers B.V. (North Holland), IFIP, 1989 